\title{
Predicting intervention effect for COVID-19 in Japan: state space modeling approach
}

\author{
Genya Kobayashi, ${ }^{1, *}$, Shonosuke Sugasawa ${ }^{2}$, Hiromasa Tamae ${ }^{3}$, Takayuki Ozu ${ }^{3}$ \\ ${ }^{1}$ Graduate School of Social Sciences, Chiba University. Chiba, Japan; \\ ${ }^{2}$ Center for Spatial Information Science, The University of Tokyo. Chiba, Japan; \\ ${ }^{3}$ Nospare Inc. Tokyo, Japan.
}

SUMMARY Japan has observed a surge in the number of confirmed cases of the coronavirus disease (COVID-19) that has caused a serious impact on the society especially after the declaration of the state of emergency on April 7, 2020. This study analyzes the real time data from March 1 to April 22, 2020 by adopting a sophisticated statistical modeling based on the state space model combined with the well-known susceptible-infected-recovered (SIR) model. The model estimation and forecasting are conducted using the Bayesian methodology. The present study provides the parameter estimates of the unknown parameters that critically determine the epidemic process derived from the SIR model and prediction of the future transition of the infectious proportion including the size and timing of the epidemic peak with the prediction intervals that naturally accounts for the uncertainty. Even though the epidemic appears to be settling down during this intervention period, the prediction results under various scenarios using the data up to May 18 reveal that the temporary reduction in the infection rate would still result in a delayed the epidemic peak unless the long-term reproduction number is controlled.

Keywords COVID-19, epidemic peak, SIR model

\section{Introduction}

Since the first case of the coronavirus disease 2019 (COVID-19) in Japan was confirmed on January 15,2020 , the number of confirmed cases has been increasing day by day. Although the Japanese government declared a state of emergency on April 7 , it does not have a legal force to regulate individual activities and remains at only requesting the avoidance of outings. Although the spread of the epidemic seems to have slowed down, one still needs to remain cautious. Needless to say, Japanese economy has been seriously shocked and the public interest mainly lies on how the number of infected persons transits in the future and when the outbreak will converge. Although there already exists a rapidly increasing number of statistical analyses of the epidemic, the statistical evidence focusing on the situations in Japan is still limited except for (1$3)$. Therefore, the purpose of this study is to provide a statistical evidence regarding the future transition of the infectious proportion in Japan, including the intensity and timing of the epidemic peak, based on the real-time data on the cumulative number of confirmed, recovered and deceased persons, shown in Figure 1. Specifically, the observations up to about two weeks after the state was declared (left panel of Figure 1) is first used to prediction under the various scenarios for the effect and length of the intervention. Then using the extended data that includes more recent observations (right panel of Figure 1), the prediction results are validated.

We consider the famous susceptible-infectedrecovered (SIR) model (4) for modeling the epidemic process as widely adopted in the existing literature on COVID-19. However, this deterministic model is not necessarily sufficient to explain the variability of the transition since the observed number is subject to nonignorable randomness. To handle such randomness in the data, we employ the state spate models combined with the SIR model (SS-SIR model) developed by $(5,6)$. The model was originally proposed for statistical modeling of the seasonal trend of influenza. The advantages of the SS-SIR model are mainly three points; (i) the unknown parameters in the SIR model can be estimated with little knowledge about the true values by adequately using the data information; (ii) future prediction of a variety of quantities such as the number of infections or the epidemic peak as well as uncertainty quantification of the prediction can be carried out easily; 

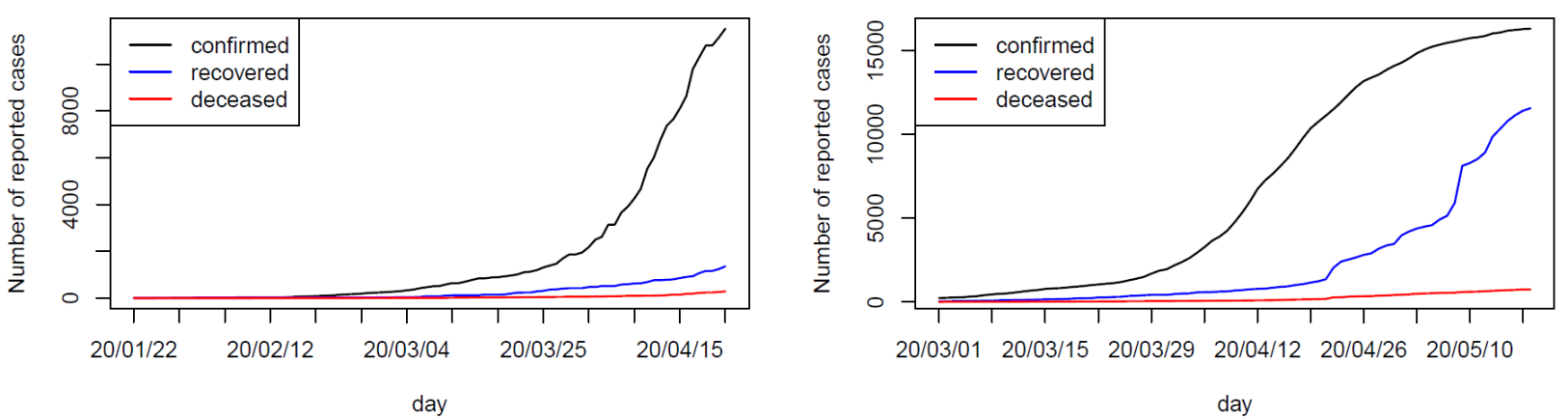

Figure 1. The cumulative numbers of confirmed, recovered and deceased persons in Japan from March 1, 2020 to April 22 (left) and to May 18 (right).

(iii) whether the real-time data follows the assumed SIR model or not can be assessed through the parameter estimate; ( $i v)$ the effect of the intervention can be estimated by appropriately extending the model. These advantages are quite essential because $(i)$ information required for modeling the epidemic trend of a new virus is scarce, (ii) it is important to compute not only point prediction but also interval prediction to understand the possible worst and best scenarios of future transition, and (iii) understanding if the real-time data actually follows the SIR model is critical for the reliability of future simulations based on the SIR model.

\section{Methods}

\subsection{Data}

We use the numbers of confirmed, recovered and deceased persons collected on an open source platform (https://www.kaggle.com/lisphilar/covid19-dataset-injapan). Although the original data starts from February 6 , the numbers before the end of February are treated collectively. This is because the confirmed numbers in this period are relatively small and using the data from March 2020 would be useful to reliably predict the future numbers of infectious persons after May 2020. Hence, the period of the data used in our analysis consists of $T=53$ days from March 1 to April 22. We use the difference between the cumulative numbers of confirmed persons and recovered plus deceased persons, denoted by $Z(t)$ for $t=1, \ldots, T$, which can be interpreted as the number of confirmed persons being infectious. It is further assumed that only $p(0<p \leq 1)$ fraction of infectious individuals can be identified by diagnosis, which is called identification rate hereafter. Then we define $Y(t)$ as $Z(t)=N p \times Y(t)$ where $N=1.265 \times 10^{8}$ is the population of Japan, thereby $Y(t)$ is the proportion of the infectious population at time $t$. Regarding the specific values of $p$, we follow the discussion in (1). Since (7) reported that 77 persons were confirmed among the possible 940 infected population, the $95 \%$ confidence interval of $p$ is $(0.059,0.105)$. Based on this argument, the results under the following three scenarios $p=0.05,0.1$ and 0.2 are compared.

\subsection{Statistical model}

Here the model proposed by ( 6 ) is described. Let $S(t)$, $I(t)$ and $R(t)$ denote the proportions of individuals being susceptible, infected and recovered population at the time $t$, respectively, satisfying $S(t)+I(t)+R(t)=1$. The SIR model describes the epidemic over time via the nonlinear ordinary differential equations (ODE) given by

$$
\begin{aligned}
& S^{\prime}(t)=-\beta S(t) I(t), \\
& I^{\prime}(t)=\beta S(t) I(t)-\gamma I(t), \\
& R^{\prime}(t)=\gamma I(t),
\end{aligned}
$$

where the unknown infection rate $\beta>0$ and removal rate $\gamma>0$ control the transition from one compartment to the next and jointly determine the epidemic process. Let $\theta(t)$ $=(S(t), I(t), R(t))$ define the three-dimensional vector of the unobserved true proportion at the time $t$. To allow randomness in the evolution of $\theta(t)$, the following model is considered:

$$
\theta(t) \mid \theta(t-1) \sim \operatorname{Dir}(\kappa f(\theta(t-1) ; \beta, \gamma)), t=1, \ldots, T,
$$

where $\operatorname{Dir}(\cdot)$ denotes the Dirichlet distribution, $f(\theta(t-1)$; $\beta, \gamma)$ is the solution of the deterministic SIR model Eq.(1) starting the ODE at $\theta(t-1)$ and $\kappa>0$ is the unknown parameter controlling the randomness in the evolution. In the above model, the conditional expectation of $\theta(t)$ given the previous state $\theta(t-1)$ is $f(\theta(t-1) ; \beta, \gamma)$, so the distribution of $\theta(t)$ is centered around the deterministic model Eq.(1). It is noted that the conditional variance of $\theta(t)$ decreases as $\kappa$ increases, thus the validity of the assumption of the deterministic model Eq.(1) can be verified through the estimate of $\kappa$.

Let $Y(t)$ be the observed value of $I(t)$. Since $Y(t)$ is not necessarily equal to the true $I(t), Y(t)$ is observed based on the following probabilistic model:

$$
Y(t) \mid I(t) \sim \operatorname{Beta}(\lambda I(t), \lambda(1-I(t))), \quad t=1, \ldots, T,
$$

where $\operatorname{Beta}(\cdot, \cdot)$ denotes the Beta distribution and $\lambda$ 
$>0$ is an unknown parameter having a similar role to $\kappa$ in Eq.(2). The statistical model for $Y(t)$ with the combination of Eq.(2) and (3) is seen as a state space model.

The unknown parameters in the model are the two parameters $\beta$ and $\gamma$ in the SIR model and two scale parameters $\kappa$ and $\lambda$ that control the randomness in the two equations Eq.(2) and (3). The estimation of these parameters and future prediction is conducted within the Bayesian framework in which we assign prior distributions for these parameters and compute the posterior distribution via the Bayes rule. Due to the complexity of the model, the analytical derivation of the posterior distribution is not feasible. Instead, we rely on the simulation-based method known as Markov Chain Monte Carlo (MCMC) algorithm (8) to generate random numbers from the posterior distribution. Then the parameter estimates are calculated and future prediction is carried out based on the output of the MCMC algorithm.

Regarding the prior distributions, we assign slightly non-informative priors to reflect the uncertainty about the new epidemic and let the data tell the truth adequately. The details of the settings of the prior distributions and algorithm are provided in Supplementary Material (http:// www.biosciencetrends.com/action/getSupplementalData. php? ID=67).

\section{Results}

\subsection{Prediction of epidemic peak}

The SS-SIR model is applied to the Japanese data with the three identification rates $p$. First, we found that the estimates of the precision parameters $\kappa$ and $\lambda$ are very large. For example, the point estimates are $\widehat{\lambda}=1.76 \times$ $10^{5}$ and $\widehat{\kappa}=3.00 \times 10^{5}$ for $p=0.1$ indicating that the deterministic SIR model explains the transition of the real-time data well. Table 1 reports the estimates and $95 \%$ credible intervals of the representative parameters. Under the three settings for $p$, the point estimates of $\beta$ are between 0.20 and 0.25 and those of $\gamma$ are between 0.13 and 0.17 . The estimates of the basic reproduction number $R_{0}$ are between 1.42 and 1.49 . For $p=0.1$, for example, the $95 \%$ credible interval of $R_{0}$ is (1.20-1.65). The estimates of PI and PT appear to vary depending on the identification rate. Figure 2 reports the future predictions of the proportion of the infectious proportion under the three identification rates. The figure allows us to easily understand the degree of uncertainty in prediction, and worst and best scenarios for the future epidemic process through the interval prediction. It is seen that the predicted timing of the epidemic peak and peak intensity depend on the identification rate through the differences in the estimates of PT and PI. Specifically, the point predictions of the trajectory of the infectious proportion have the timing of the peak on July 19, July 21 and August 1 with the intensities and $95 \%$ prediction intervals of $3.84 \%(1.23 \%-7.37 \%)$, $2.60 \%(0.42 \%-6.40 \%)$ and $2.25 \%(0.19 \%-6.14 \%)$ for $p=0.05,0.1$ and 0.2 , respectively. The sensitivity of prediction results with respect to $\mathrm{p}$ was also found in (1), but that under our setting of $p$ is far less dramatic. Moreover, all the scenarios predict that the epidemic

Table 1. Estimates and $95 \%$ credible intervals of parameters of the SS-SIR model under the three identification rates $p$.

\begin{tabular}{llccc}
\hline & & & Estimate $(95 \%$ interval) \\
\hline Parameter & Description & $p=0.05$ & $p=0.1$ & $p=0.2$ \\
$\beta$ & Infection rate & $0.20(0.12-0.32)$ & $0.25(0.13-0.47)$ & $0.25(0.14-0.53)$ \\
$\gamma$ & Removal rate & $0.13(0.07-0.24)$ & $0.17(0.09-0.39)$ & $0.17(0.09-0.44)$ \\
$R_{0}(=\beta / \gamma)$ & Basic reproduction number & $1.49(1.30-1.70)$ & $1.42(1.20-1.65)$ & $1.42(1.19-1.65)$ \\
PT & Peak timing & $156(105-249)$ & $151(96-255)$ & $160(97-261)$ \\
PI $(\%)$ & Peak intensity & $4.55(1.86-7.92)$ & $3.46(0.77-7.20)$ & $3.34(0.68-7.22)$ \\
\hline
\end{tabular}

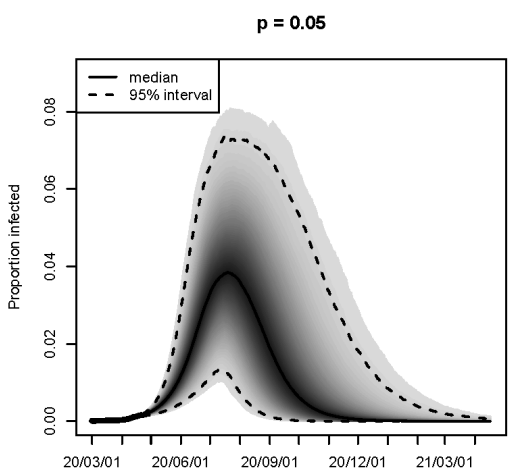

day

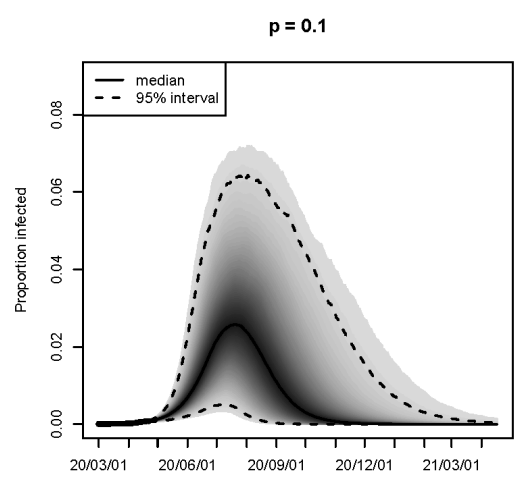

day

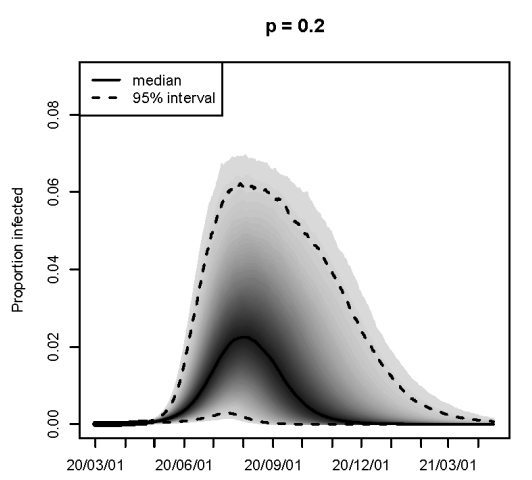

day

Figure 2. Results of the prediction of the proportion of the infectious population with $p=0.05$ (left), 0.1 (center) and 0.2 (right). The observed data points $\{Y(t), t=1, \ldots, T\}$ are shown by the black circles. 
peak comes during the summer 2020. This result is also consistent with (1).

\subsection{Effect of intervention}

On April 7, 2020, the Japanese government declared a state of emergency aiming at reducing human contacts by $80 \%$, which is considered to be sufficient to terminate the epidemic. However, the government reports that the actual reduction is still limited to around $60 \%$ or $70 \%$ (https://corona.go.jp), mainly because the state does not have a legal force to regulate individual activities. Also, the Japanese government was initially planning to lift the state on May 6, but the public concern lay on whether such a short period of the state of emergency is sufficient or not.

Through simulation, we here assess the efficacy of further intervention and public awareness on mitigating the infection risk under various scenarios. Specifically, we consider various settings for the degrees of reduction in human contacts that are achieved by the government during the intervention and by the public awareness after the intervention, and the period of intervention denoted by $T^{*}$, under the state of emergency and predict the future epidemic transitions. Here, we focus on $p$ $=0.1$. The results under $p=0.05$ and 0.2 are found in Supplementary Material (http://www.biosciencetrends. com/action/getSupplementalData.php? ID=67). It is recognized that the realization of the effect of reducing human contacts takes about two weeks since the incubation period of COVID-19 is at most 2 weeks as reported by World Health Organization. Since April 22, the last date in the real-time data, is almost two weeks after the declaration of the state of emergency, we assume that the infection rate changes from $\beta$ to $c \beta$ from April 23. For the degree of reduction in human contacts, the following six scenarios are considered: $c=0.6,0.5$, $0.4,0.3,0.2$ and 0.1 If $80 \%$ reduction of human contacts was achieved, the reality would have corresponded to $c=$ 0.2 or 0.1 . In view of the current situation, however, $c=$ 0.4 or 0.3 would be closer to the reality. We also suppose that the intervention will continue for $T^{*}$ days from April 23 with the three scenarios, $T^{*}=14,28$ and 45 . Note that $T^{*}=14$ corresponds to May 6 on which the government was initially planning to lift the state. The other two dates to respectively correspond to the two-week and onemonth extension of the intervention that continue until May 20 and June 6, respectively. We further suppose that the infection rate becomes $c^{*}$ after the intervention period with the three scenarios: $c^{*}=1,0.9$ and 0.8 . The first scenario implies that the level of human mobility after the intervention returns to the original level before the intervention. The latter two scenario can reflect the remaining strain in the public awareness on mitigating the spread of infection through, for example, voluntary avoidance of outings and social distancing.

Figure 3 presents the nine panels on the future prediction under the combinations of the three scenarios of each $T^{*}$ and $c^{*}$. Comparing the different scenarios of $T^{*}$, the figure reveals that setting $\mathrm{c}$ to smaller values is effective only when it is combined with larger $T^{*}$. For example, the left upper panel of Figure 3 exhibits little differences among the six choices of $\mathrm{c}$ when $c^{*}=1$ and $T^{*}=14$. Contrary, the small values of $c$ such as $c=0.2$ with $T^{*}=28$ and 45 can lead to a convergence of the epidemic. Under $c=0.2$ and $c^{*}=1$, the epidemic can be terminated in terms of point prediction when $T^{*}=45$, while the epidemic peak belatedly comes on September 3, 2020 with $2.2 \%$ of the peak intensity when $T^{*}=14$. The result suggests that the termination of the intervention due to the initial plan of lifting the state of emergency on May 6 would have been too early and only resulted in a slight delay in the epidemic peak and a slight reduction in the peak intensity.

The degree of reduction in $\beta$ after the intervention, $c^{*}$, also has a dramatic effect on the consequence of the epidemic. The upper panels of Figure 3 show that the efficacy of the temporary reduction in $\beta$ under the intervention can be quite limited if $\beta$ returns to the original level after the intervention. In contrast, if at least $20 \%$ reduction in $\beta$ can be achieved for a sufficiently long period of time after the intervention, the epidemic can be effectively suppressed. In the case of $c^{*}=0.9$, for example, the peak intensity is more than halved to $1.11 \%$ with the peak on September 13 even under the mild degree of intervention for a short period of time $(c$ $=0.6$ and $\left.T^{*}=14\right)$. When a longer intervention $T^{*}=45$ is carried out, the peak is further delayed to November 11 with $0.81 \%$ of the peak intensity. Furthermore, in the case of $c^{*}=0.8$, the figure shows the epidemic is almost completely suppressed in terms of point prediction regardless of the degree of intervention and length of intervention period. This would be because that the infectious proportion decreases during the intervention and the reproduction number under $c^{*}=0.8$ remains close to one even after the intervention. To summarize, our results show that not only the degree of reduction in $\beta$ during the intervention but also and more importantly the length of intervention and the long-term level of $\beta$ after the intervention is critical to control the spread of the epidemic.

Figure 4 presents a more detailed picture of the prediction results around the dates indicated by $T^{*}$. The figure shows that there are scenarios under which the proportion of the infectious population comes to increase again as the infection rate changes from $c \beta$ to $c^{*}$. Whether there will be another wave generally depends on the combination of $c, T^{*}$ and $c^{*}$, but there most likely would have been to see a rise in the infectious proportion in the case of $T^{*}=14$. In the figure, the more recent observations between April 23 and May 18 are also plotted. The figure shows that the predicted trajectories do not seem to follow well the stationary and reduction in the infectious proportion due to the sudden change 
in the infection rate under the scenarios. It seems that the decline in the trajectories in the case of $c=0.6$ appear to resemble the actual rate at which the observed infectious proportion decreased. In the following section, a modified model is estimated using an extended dataset to validate our prediction method.

\subsection{Validating the prediction results}

As requested by one of the reviewers, the dataset is updated to include more recent observations. Given that the unsettled epidemic situation, the Japanese government has decided to postpone the lifting of the state until the end of May $(T=79)$. On May 14 , the state was partially lifted for the 39 prefectures among out of where the situation is somewhat milder. For the remaining 8 prefectures, the government is planning to lift the state by the end of May. Using the updated dataset, the effect of the intervention can be estimated based on a modified version of the model Eq.(2) and the foregoing prediction results can be validated. Based on the current plan of the government, the infection rate $\beta$ is assumed to become $c \beta, 0<c<1$, from April 23 as in Section 3.2, but now $c$ is estimated from the data. The estimate of $c$ stands for the effect of the intervention under the state of emergency.

For $p=0.1$, the point estimate of $c$ is 0.583 with the $95 \%$ credible interval $(0.307-0.787)$. Therefore, this

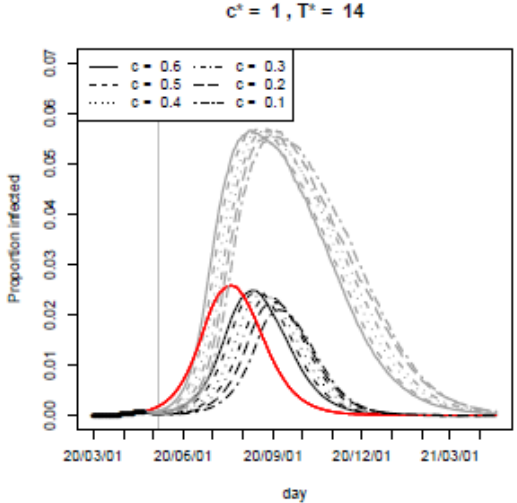

day

$\mathrm{c}^{\star}=0.9, \mathrm{~T}^{\star}=14$

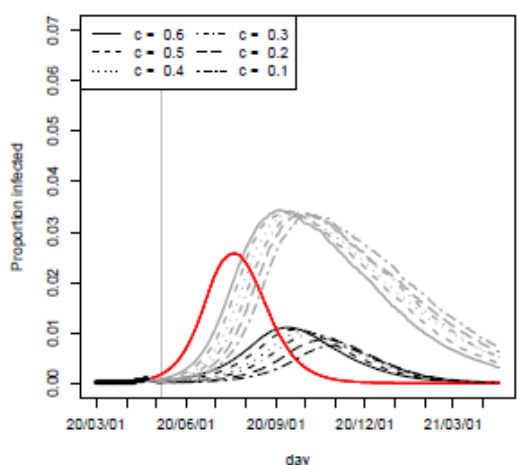

$\mathrm{c}^{\star}=0.8, \mathrm{~T}^{\star}=14$

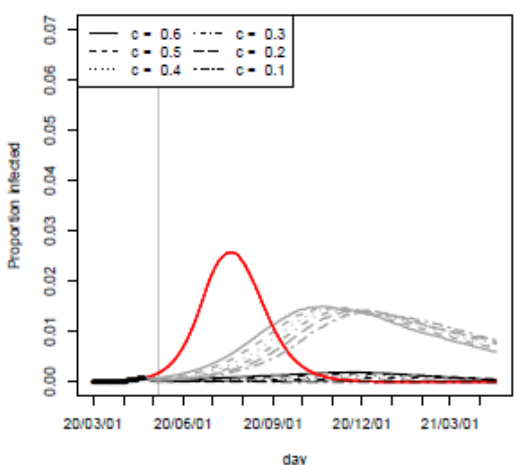

$c^{\star}=1, T^{\star}=28$

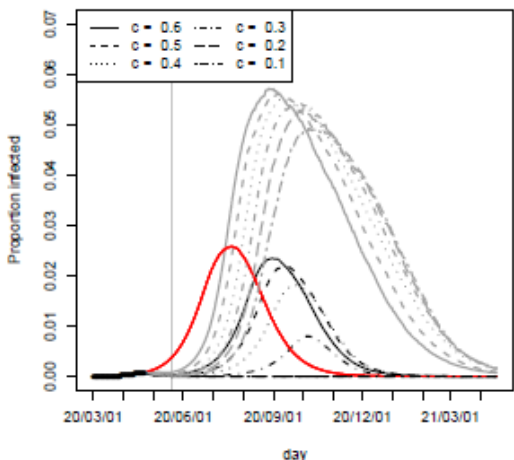

$\mathrm{c}^{*}=0.9, \mathrm{~T}^{*}=28$

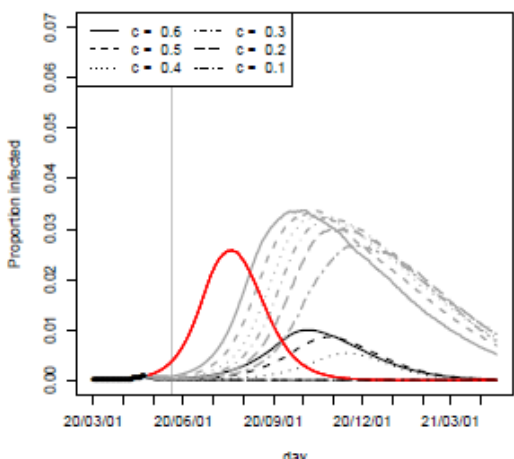

$\mathrm{c}^{*}=0.8, \mathrm{~T}^{*}=28$

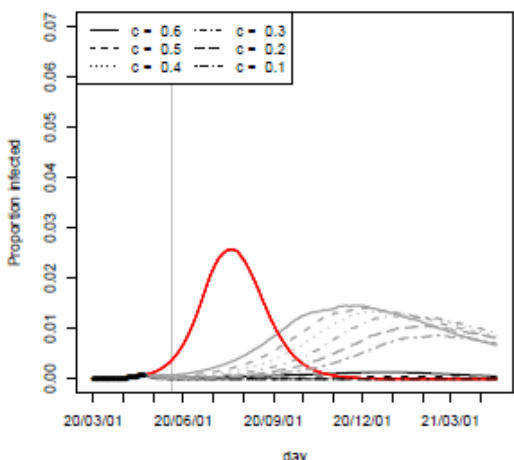

$c^{*}=1, T^{*}=45$

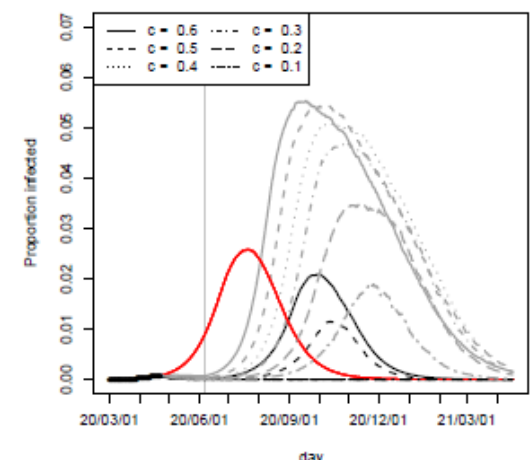

$\mathrm{c}^{*}=0.9, \mathrm{~T}^{*}=45$

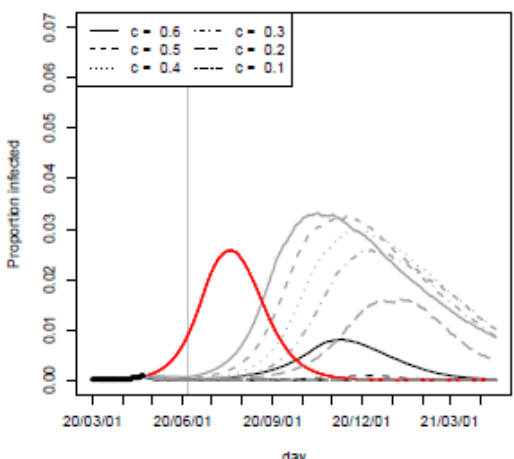

$\mathrm{c}^{*}=0.8, \mathrm{~T}^{*}=45$

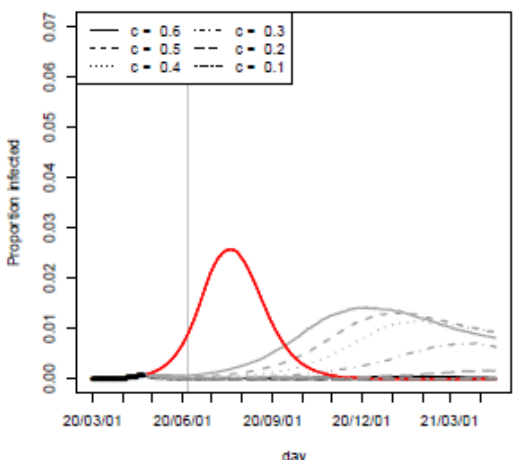

Figure 3. Future prediction under the nine combinations of $T^{*}$ (the period of the intervention) and $c^{*}$ (the multiplier for $\beta$ after the intervention) for $\boldsymbol{p}=\mathbf{0 . 1}$. The red, black and grey curves respectively represent the future point prediction without intervention shown in Figure 2 , point prediction under each scenario and upper bounds of the $95 \%$ prediction intervals. The black circles represent the observed data points. The grey vertical lines indicate the dates on which there is a change in the infection rate represented by $c^{*}$. 


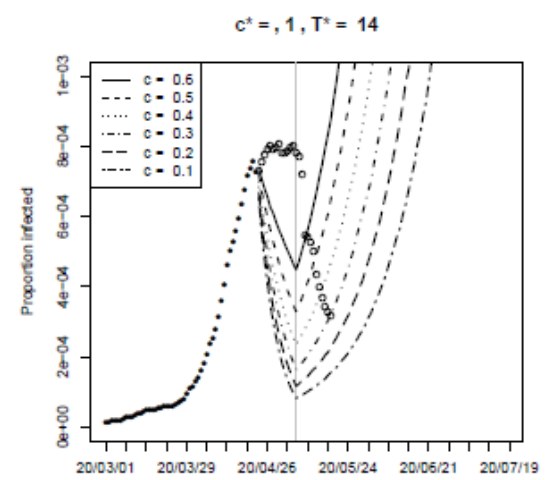

day

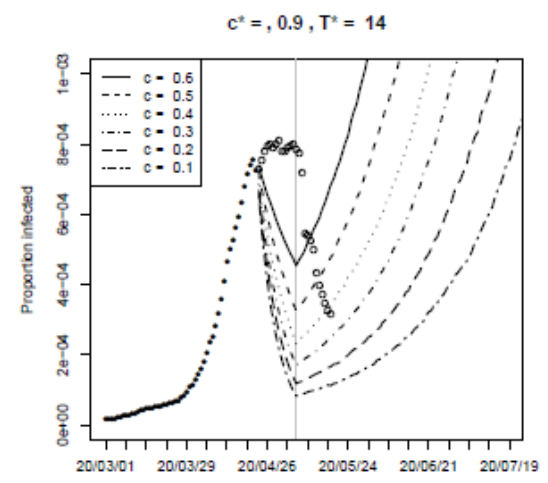

day

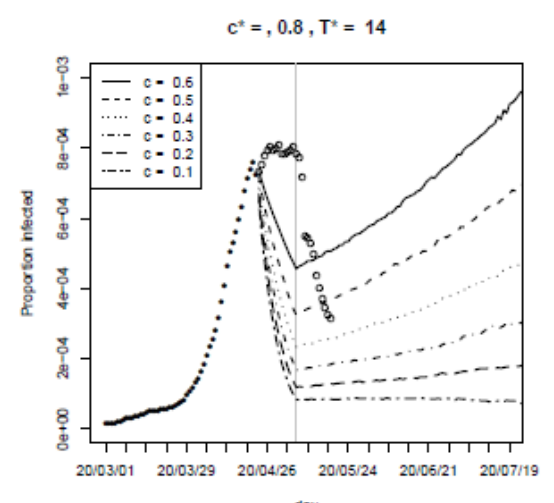

day

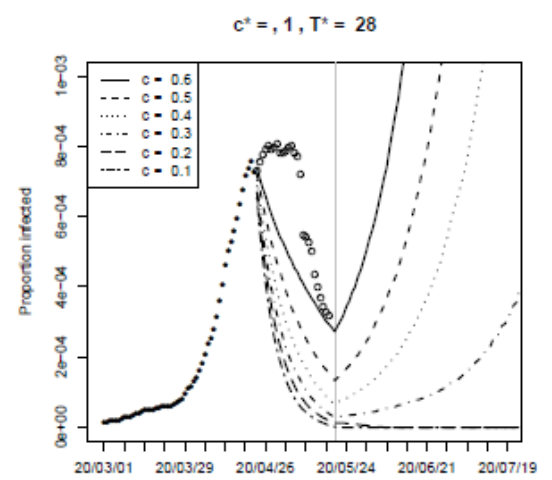

day

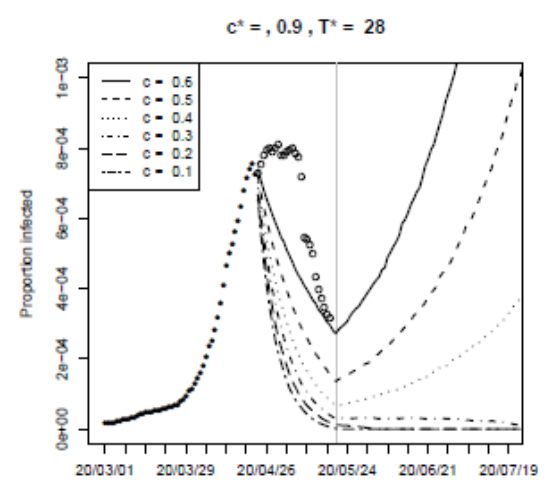

day

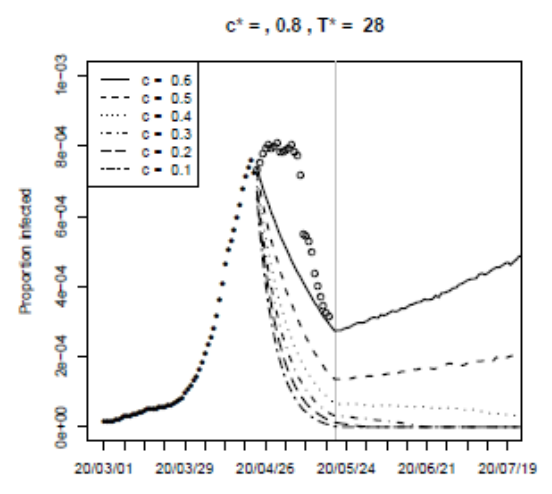

day

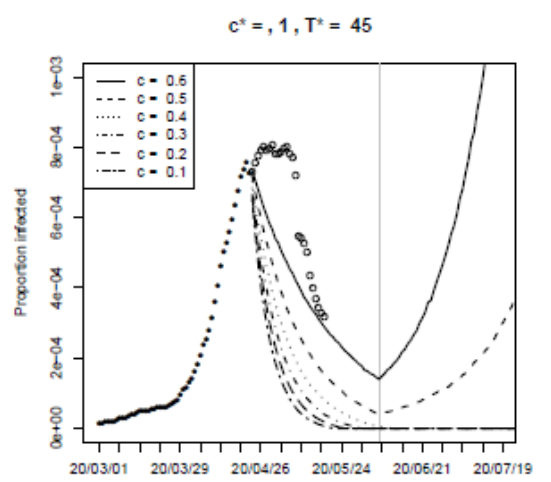

day

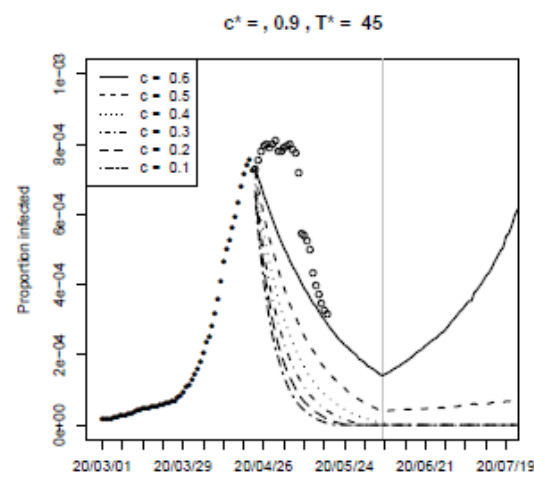

day

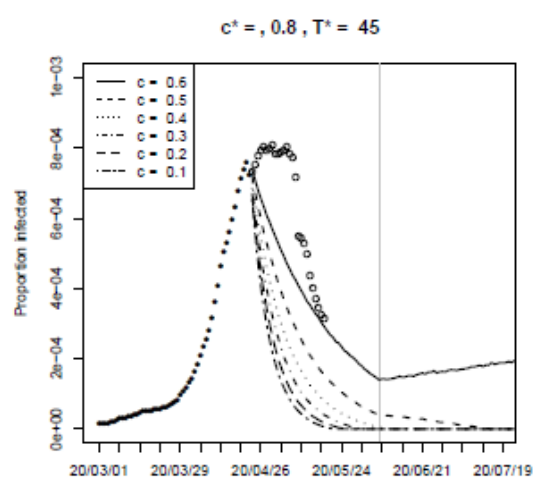

day

Figure 4. Details Figure 3. around the dates on which the infection rate changes according to $\boldsymbol{c}^{*}$. The curves represent the future point prediction. The black circles are the observed data points up to April 22 and white circles are the observed data points additionally obtained after April 22 up to May 18. The grey vertical lines indicate the dates on which there is a change in the infection rate represented by $c^{*}$.

estimate is consistent with the conjecture in Section 3.2 that the actual reduction in human contact under the intervention is by about $40 \%$. The estimate for $R_{0}$ is 1.482 (1.273-1.723). Our estimate for $R_{0}$ remains unchanged even when the data include more recent observations. The estimate of the effective reproduction number $c R_{0}$ during the intervention period is 0.854 (0.495-1.091). Although the upper bound of the $95 \%$ credible interval of $c R_{0}$ slightly exceeds one, the posterior distribution is concentrated mainly below one, implying that the epidemic would progress towards convergence if the value of $c$ continues to remain around our estimate. Some more details are found in Supplementary Material (http:// www.biosciencetrends.com/action/getSupplementalData. php?ID=67).
As in Section 3.2, the future trajectories of the infectious proportion are simulated under the following scenarios. The infection rate remains to be $c \beta$ until $T^{*}$ days from May 18 where $T^{*}=13,27,43$ and 74 , corresponding to May 31, June 14, June 30 and July 31. Then it becomes $c^{*} \beta$ with $c^{*}=1,0.9$ and 0.8 after the intervention period. Figure 5 presents the predicted infectious proportions under the various scenarios. Similar to Figure 3, a smaller $c^{*}$ combined with a larger $T^{*}$ will suppress the epidemic. While the estimate of $c^{*}$ implies that the intervention is successful to some extent, the prediction results suggest the possibilities of a second wave in the case of, for example, $c^{*}=1$ and $T^{*}=13$. Finally, Figure 6 provides a more detailed picture of Figure 5 around the dates indicated by $T^{*}$. It 

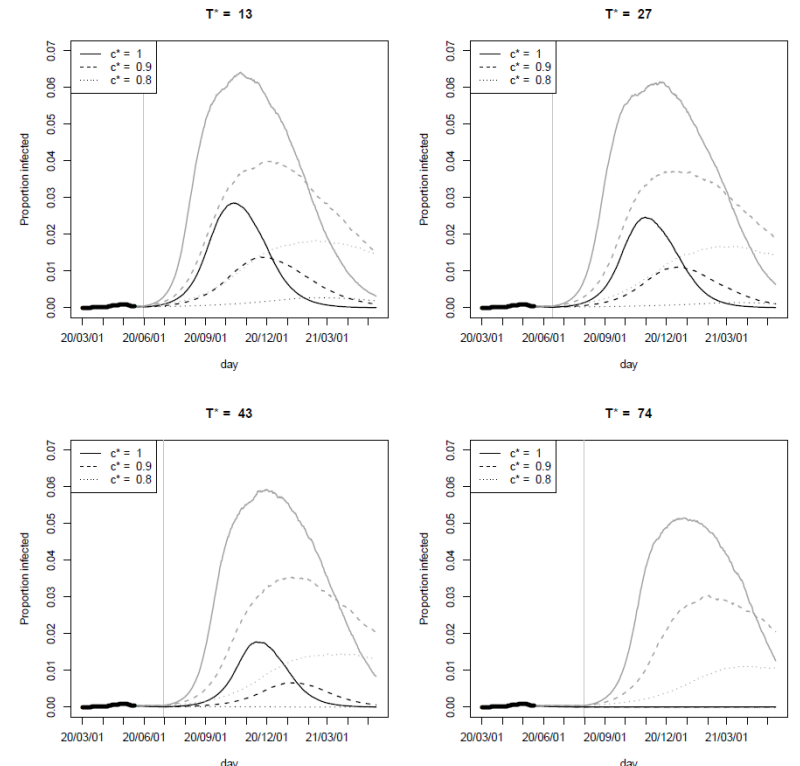

Figure 5. Future prediction under the combinations of $T^{*}$ (the period of the intervention) and $c^{*}$ (the multiplier for $\beta$ after the intervention) for $\boldsymbol{p}=0.1$ using the extended dataset. The black and grey curves respectively represent the future point prediction under each scenario and upper bounds of the $95 \%$ prediction intervals. The grey vertical lines indicate the dates on which there is a change in the infection rate represented by $c^{*}$.

is seen that the infectious proportions are successfully suppressed until the day on which $\mathrm{c}$ changes to $c^{*}$. The rate at which the infectious proportions decline naturally follows that at which the observed proportions decline. As in the previous analysis, the infectious proportions are most likely predicted to rise again after those change points. Since a further extension of the intervention does not seem very feasible under the recent decline in the newly confirmed cases and the public pressure caused by the economic crisis, controlling the post-intervention infection rate represented by $c^{*}$ is critical as in the previous prediction results.

\section{Discussion}

In this research, we have employed the probabilistic version of the famous SIR model, called SS-SIR model, to model the real-time data on the infectious population of COVID-19 in Japan. The advantage of the SS-SIR model is that we can obtain not only future point prediction but also uncertainty quantification through, for example, the future prediction intervals. The basic reproduction number $R_{0}$ is estimated to be approximately between 1.4 and 1.5 in this study. This is smaller than the estimate of 2.6 in (1) obtained from the SEIR model applied to the early stage data in Japan. Note, however, that (1) did not estimate the removal rate and onset rate but fixed their values to those found in the existing studies. We also confirmed that the estimate of $R_{0}$ remains unchanged even when the model is estimated by using the extended dataset. Moreover,
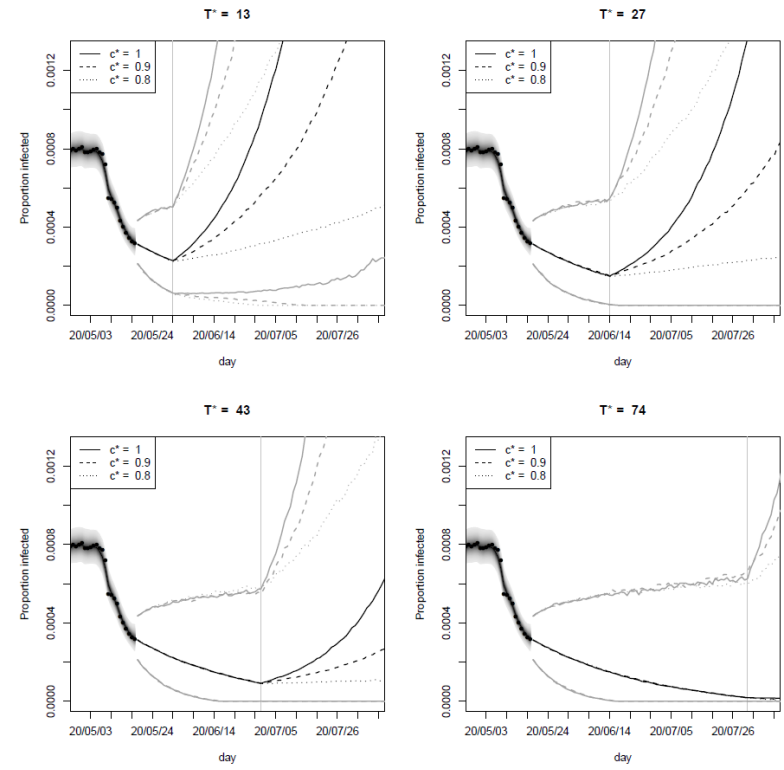

Figure 6. Details Figure 5 around the dates on which the infection rate changes according to $c^{*}$. The black and grey curves respectively represent the future point prediction and $95 \%$ prediction intervals. The black circles represent the observed data points along with the $95 \%$ credible intervals. The grey vertical lines indicate the dates on which there is a change in the infection rate represented by $c^{*}$.

our estimate in the case of Japan is also smaller than those reported from the case studies in China (9-14). Our result may have reflected the fact that the number of cases in Japan does not increase as rapidly as other countries (15).

Through the future prediction under the various scenarios on the possible reduction in the infection rate $\beta$ and the length of the intervention period, we have obtained the following epidemiological insights:

- Even if a large reduction in the infection rate could be achieved during the intervention period (e.g. the state of emergency), the convergence of the epidemic can still depend on the long-term value of the infection rate $c^{*} \beta$ after the intervention.

- As long as the value of $c^{*} \beta$ can be maintained to be slightly smaller even after the intervention period than the value of $\beta$ before the intervention $\left(c^{*}<1\right)$, there is a possibility that the epidemic terminates with a significantly smaller epidemic size than the case without intervention.

- Using the extended dataset, the effect of intervention under the state is about $40 \%$ reduction in the infection rate.

These findings confirm that the intervention under the state of emergency and its extension has been successful to suppress the epidemic to some extent. Under the public pressure, the government will most likely lift the state for all prefectures by the end of May. Our findings suggest that a long-term effort to control the infection as indicated by the parameter $c^{*}$ is indispensable. 


\section{Acknowledgements}

This research was supported by Japan Society for the Promotion of Science (KAKENHI) Grant Numbers $18 \mathrm{~K} 12754$ and $18 \mathrm{~K} 12757$. This problem in the present paper was provided by Nospare Inc..

Author Contributions: H.T. and T.O. collected the data. G.K. and S.S. analyzed the empirical data using the models. All the authors participated in the study design, drafted the manuscript, and gave comments on the current version of the manuscript.

\section{References}

1. Kuniya T. Prediction of the Epidemic Peak of Coronavirus Disease in Japan, 2020. J Clin Med. 2020; 9:789.

2. Karako K, Song P, Chen Y, Tang W. Analysis of COVID-19 infection spread in Japan based on stochastic transition model. BioSci Trends. 2020; 14:134-138.

3. Mizumoto K, Kagaya K, Zarebski A, Chowell G. Estimating the asymptomatic proportion of coronavirus disease 2019 (COVID-19) cases on board the Diamond Princess cruise ship, Yokohama, Japan, 2020. Euro Surveill. 2020; 25:2000180

4. Kermack W, McKendrick A. Contribution to the Mathematical Theory of Epidemics. Proc R Soc Lond A Math Phys Sci. 1927; 115:700-721.

5. Dukic V, Lopes HF, Polson NG. Tracking Epidemics with Google Flu Trends Data and a State-Space SEIR Model. J Am Stat Assoc. 2012; 107:1410-1426.

6. Osthus D, Hickmann K, Caragea PC, Higdon D, Del Valle SY. Forecasting seasonal influenza with a state-space SIR model. Ann Appl Stat. 201; 11:202-204.

7. Bloomberg. Japan's Hokkaido may Have 940 Infected, Researcher Says. https://www.bloomberg.com/news/ articles/2020-03-03/japan-s-hokkaido-could-have-up-to- 940-infected-researcher-says (accessed March 3, 2020).

8. Gamerman D, Lopes HF. Markov Chain Monte Carlo: Stochastic Simulation for Bayesian Inference, Second Edition. Chapman and Hall/CRC. CRC Press, 2006.

9. Sun H, Qiu Y, Yan H, Huang Y, Zhu Y, Chen S. Tracking and predicting COVID-19 epidemic in China mainland. medRxiv. 2020; doi: https://doi. org/10.1101/2020.02.17.20024257

10. Imai N, Cori A, Dorigatti I, Baguelin M, Connelly C, Riley S, Ferguson N. Report 3: Transmissibility of 2019nCoV. Imperial College London: London, UK. 2020.

11. Liu Y, Gayle AA, Wilder-Smith A, Rocklov J. The reproductive number of COVID-19 is higher compared to SARS coronavirus. J Travel Med. 2020; 27 :taaa021.

12. Liu $\mathrm{T}, \mathrm{Hu} \mathrm{J}$, Hang $\mathrm{M}$, et al. Transmission dynamics of 2019 novel coronavirus (2019-nCoV). bioRxiv. 2020; doi: https://doi.org/10.1101/2020.01.25.919787

13. Tang B, Wang X, Li Q, Bragazzi N, Tang S, Xiao Y, Wu J. Estimation of the Transmission Risk of the 2019-nCoV and Its Implication for Public Health Interventions. J Clin Med. 2020; 9:462.

14. Wu J, Leung K, Leung G. Nowcasting and forecasting the potential domestic and international spread of the 2019-nCoV outbreak originating in Wuhan, China: A modelling study. Lancet 2020; 395:689-697.

15. Nippon.com. Coronavirus Cases by Country. https:// www.nippon.com/en/japan-data/h00673/coronaviruscases-by-country.html (Accessed April 24, 2020).

Received May 1, 2020; Revised May 21, 2020; Accepted May 26, 2020.

*Address correspondence to:

Genya Kobayashi, Graduate School of Social Sciences, Chiba University. 1-33, Yayoi-cho, Inage-ku, Chiba 263-8522, Japan.

E-mail: gkobayashi@chiba-u.jp

Released online in J-STAGE as advance publication May 28, 2020 . 